\title{
Lymphangioleiomyomatosis and tuberous sclerosis: Where is the border?
}

\author{
F. Bonetti*, P. Chiodera**
}

Pulmonary lymphangioleiomyomatosis (PLAM) is a rare disease, characterized by an abnormal proliferation of smooth muscle throughout the lung; it occurs exclusively in women, generally of reproductive age [1]. The muscle cells proliferating in PLAM show little or no atypia at histological level. Therefore, PLAM has been considered to be a hamartomatous lesion rather than a true neoplastic process. However, in spite of the bland cytological features, the disease causes a progressive structural remodelling of the lung, leading to serious impairment of pulmonary function. Whilst survival curves now seem better than previously believed and hormonal treatment has been introduced with encouraging results [2], some patients progress to a condition necessitating lung transplantation.

A disease with overlapping morphological features is occasionally encountered within the neurological syndrome of tuberous sclerosis (TS), also called Bourneville's disease. Indeed, the first description of PLAM, in 1918, was by LAUTENBACHER [3], in a female patient with the stigmata of TS. Subsequently, various authors have reported examples of PLAM, in patients without clinical evidence of TS. The question of whether PLAM is a "forme fruste" of tuberous sclerosis or a distinct disease has since been debated.

In 1975, CORRIN et al. [1] reviewed 34 cases in the literature at that time and added 23 previously unpublished cases. In this work, the authors discussed the possibility of a relationship between tuberous sclerosis and PLAM, concluding that the question must be considered to be unresolved.

When we consider the clinical settings of PLAM and TS, important differences emerge. Notably, PLAM develops exclusively in females, whilst TS affects both sexes (but lung lesions in TS develop almost exclusively in females), and familial history is common in TS patients, whilst it is absent in PLAM. These findings led STOvin et al. [4] to conclude that "lymphangiomyomatosis and tuberous sclerosis are probably not different aspects of the same disease process but seem to be two different disorders". Other authors have expressed an opposite view, as in a recent paper by CASTRO et al. [5] reporting the clinical features of nine TS patients with pulmonary involvement. The authors concluded that "... TS is similar to PLAM in terms of its natural history, clinical features and response to treatment".

*Istituto di Anatomia Patologica, Università di Verona, Italy and **Servizio di Anatomia Patologica, Ospedale di Esine (Brescia), Italy.
The key to addressing this unresolved question seems to be the definition of the diagnostic criteria of TS. The classical Vogt triad (seizures, mental retardation and facial angiofibroma) was the first important attempt to clinically define the syndrome. This diagnostic triad was universally accepted and was, for a long time, considered the hallmark of the disease. Whilst useful for clinicians, this approach led to a delay in the recognition of other diagnostic signs of TS, particularly the presence and classification of visceral lesions, such as angiomyolipoma, so characteristic in this hereditary disease of autosomal dominant transmission. With the accumulation of knowledge on TS and the introduction of new diagnostic techniques, the Vogt triad has lost much of its importance. The Vogt's diagnostic triad is now considered obsolete and too strict to encompass all cases of TS.

GOMEz [6] revised the criteria for diagnosis of TS, showing that TS is much more common than previously estimated, and that only $29 \%$ of cases had the complete Vogt's triad. A detailed analysis of the hierarchy of clinical and imaging features necessary for a definitive, presumptive or suspect diagnosis of TS can be found in the work of Gomez [6]. In this revised classification of diagnostic criteria, PLAM is considered sufficient for a presumptive diagnosis of TS, when alone; and sufficient for a definitive diagnosis when in combination with at least one other feature of the same hierarchical diagnostic level (such as renal angiomyolipoma).

TS is, by definition, a disease with multiorgan involvement. Most, if not all organs, can present abnormal growths of hamartomatous/neoplastic nature, thus leading to a great variety of signs and symptoms. The protean manifestations of TS account for the difficulties in establishing diagnostic criteria.

It has always been difficult to place PLAM in the context of TS. In fact, PLAM has most often been observed as an isolated disease in patients without other symptoms or signs characteristic of TS. In the work of CORRIN et al. [1], only 2 of the 28 cases evaluated had evidence of renal angiomyolipoma. At that time, however, most cases could not be thoroughly studied with modern imaging techniques.

In the interesting study by MAZIAK et al. [7] published in this issue of the Journal, the authors show a much stronger association between PLAM and renal angiomyolipoma than previously believed. Eight of 14 patients (57\%) had renal tumours consistent with angiomyolipomas, five 
of which were bilateral. It should be noted that, when the revised diagnostic criteria of GoMEz [6] for TS are applied, the majority of the patients reported by MAZIAK et al. [7] could be definitely diagnosed as TS.

In addition, one patient showed a nodular growth in the pancreas, which was histologically proven to be an angiomyolipoma. Angiomyolipoma is not restricted to the kidney and has been reported in a number of locations; however, this is the first report of a similar lesion in the pancreas.

The possible cause of this surprisingly high association is well delineated by the authors. Prolonged survival and extensive radiological examination seem to be the key factors. Whilst PLAM manifests through often dramatic symptoms, angiomyolipoma both renal and extrarenal are often asymptomatic, do not usually require surgical treatment and may go undocumented. Today, clinicoradiological investigation and fine needle aspiration cytology can conclusively diagnose angiomyolipoma preoperatively in most cases [8]. Only when present in unusual locations, such as the pancreas, angiomyolipoma can pose difficult diagnostic problems, thus leading to surgery. This has been the case both in the patient reported here and in a patient who recently came to our observation [9].

The findings of MAZIAK et al. [7] add further and significant evidence of the clinical relationship between PLAM and angiomyolipoma. This strict correlation has recently also been demonstrated at the cellular level. In fact, immunohistochemical studies have shown that the muscle cells of PLAM exhibit a peculiar and distinctive phenotype. These cells are positive not only for actin and desmin (the typical markers of muscle nature) but also for a melanogenesis marker, such as HMB45 [10, 11]. Extensive studies have shown that this phenotype is indeed characteristic of PLAM: a number of other muscle proliferations, both in the lung and in other organs, have been tested with the same markers and none has shown the same immunophenotypical pattern [12, 13].

Thus, the coexpression of muscle and melanogenesis markers has been used to identify PLAM on bioptic specimens, particularly on transbronchial biopsy, and distinguish PLAM from other morphologically similar proliferations (e.g. Kaposi's sarcoma, focal muscle hyperplasia) [13]. Interestingly, this peculiar muscle cell, to identify which we have proposed the descriptive term of "perivascular epithelioid cell" (PEC) [14], is also present in angiomyolipoma of the kidney $[12,15]$. Thus, PLAM and angiomyolipoma are indeed closely related, not only clinically but also in their distinctive cellular component.

Whilst these data strongly indicate a closer relationship between PLAM and TS than previously believed, only genetic studies will definitively clarify their relationship. A similar problem is encountered with angiomyolipoma. Whilst the presence of multiple angiomyolipomas in a patient with evidence of TS is easily classified, the true significance of the occurrence of these lesions in patients without TS (sporadic angiomyolipoma) is not well-known, in particular whether they are related to the same genetic defect. Our knowledge of the genetic defects underlying TS, has rapidly evolved over recent years.
Genetic studies of TS have shown the presence of heterogeneity and two disease-determining genes have now identified: TSC1 and TSC2. TSC1 has been identified on chromosome 9 (9p34.3) [16], and TSC2 on chromosome 16 [17].

Recently, the European Chromosome 16 Tuberous Sclerosis Consortium has identified and characterized the TSC2 on chromosome 16 (16p13.3) [18]. Loss of heterozygosity on chromosome $16 \mathrm{p} 13.3$ in angiomyolipomas from tuberous sclerosis patients has recently been reported, using the polymerase chain reaction from histological sections [19]. The same genetic defect has also been observed in cases of angiomyolipoma in patients without evidence of TS. A similar study needs to be performed on PLAM both from TS patients and from sporadic cases. Technical problems make the genetic study of PLAM more difficult.

In fact, whilst angiomyolipoma is a nodular tumourlike proliferation distinct from the surrounding kidney, PLAM is a subtle proliferation of muscle cells within the normal structures of the lung. Isolation of a pure population of cells is of major importance for genetic studies. However these problems will probably be overcome by isolating nodules of PLAM through microdissection techniques.

It seems reasonable to consider that genetic analysis of PLAM will, in the near future, indicate whether the same genetic defect is present in the TS-associated lesions and in PLAM. In the meantime, we cannot but agree with the conclusion of MAZIAK et al. [7] that there is increased evidence that "... PLAM and tuberous sclerosis represent a spectrum of a similar disease process". TS should be suspected and searched for in all patients with PLAM; the border separating these two entities has become rather indistinct.

\section{References}

1. Corrin B, Liebow AA, Friedman PJ. Pulmonary lymphangiomyomatosis: a Review. Am J Pathol 1975; 79: 348-382.

2. Taylor JR, Ryu J, Colby TV, Raffin TV. Lymphangioleiomyomatosis: clinical course in 32 patients. N Engl J Med 1990; 323: 1254-1260.

3. Lautenbacher R. Dysembriomes métotipiques des reins, carcinose submiliere aigùe poumon avec amphysème généralisé et double pneumothorax. Ann Méd Interné (Paris) 1918; 5: 435-450.

4. Stovin PGI, Lum LC, Flower CDR, Darke CS, Beeley $\mathrm{M}$. The lungs in lymphangiomyomatosis and in tuberous sclerosis. Thorax 1975; 30: 497-509.

5. Castro M, Shepherd CW, Gomez MR, Lie JT, Tyu JH. Pulmonary tuberous sclerosis. Chest 1995; 107: 189195.

6. Gomez MR. Phenotypes of the tuberous sclerosis complex with a revision of diagnostic criteria. In: Johnson WG, Gomez MR, eds. Tuberous Sclerosis and Allied Disorders. New York, NY, The New York Academy of Sciences, 1991; pp. 1-7.

7. Maziak DE, Kesten S, Rappaport DC, Maurer J. Extrathoracic angiomyolipoma in lymphangioleiomyomatosis. Eur Respir J 1996; 9: 402-405. 
8. Bonzanini M, Pea M, Martignoni G, et al. Preoperative diagnosis of renal angiomyolipoma: fine needle aspiration cytology and immunocytochemical characterization. Pathology 1994; 26: 170.

9. Zamboni G, Pea M, Martignoni G, et al. Clear cell "sugar" tumor of the pancreas: a novel member of the family of lesions characterized by the presence of perivascular epithelioid cells (PEC). Am J Surg Pathol; (in press).

10. Gown A, Vogel A, Hoak D, et al. Monoclonal antibodies specific for melanocytic tumors distinguish subpopulations of melanocytes. Am J Pathol 1986; 123: 195-203.

11. Bacchi CE, Bonetti F, Pea M, Martignoni G, Gown AM. HMB45: a review. Appl Immunohistochem; (in press).

12. Pea M, Bonetti F, Zamboni G, et al. Melanocyte marker HMB-45 is regularly expressed in angiomyolipoma of the kidney. Pathology 1991; 23: 185-188.

13. Bonetti F, Chiodera P, Pea M, et al. Transbronchial biopsy in lymphangiomyomatosis of the lung: HMB45 for diagnosis. Am J Surg Pathol 1993; 17(11): 1092-1102.
14. Bonetti F, Pea M, Martignoni G, Zamboni G. PEC and sugar. Am J Surg Pathol 1992; 16(3): 307-308.

15. Chan JKC, Tsang WYW, Pau MY, Tang MC, Pang SW, Fletcher CDM. Lymphangiomyomatosis and angiomyolipoma: closely related entities characterized by hamartomatous proliferation of HMB45-positive smooth muscle. Histopathology 1993; 22: 445-455.

16. Fryer AE, Chalmers A, Connor JM, et al. Evidence that the gene for tuberous sclerosis is on chromosome 9. Lancet 1987; i: 659-661.

17. Kandt RS, Haines JL, Smith M, et al. Linkage of an important gene locus for tuberous sclerosis to a chromosome 16 marker for polycystic kidney diasease. Nature Genet 1992; 2: 37-41.

18. The European Chromosome 16 Tuberous Sclerosis Consortium. Identification and characterization of the tuberous sclerosis gene on chromosome 16. Cell 1993; 75: 1305-1315.

19. Green AJ, Smith M, Yates JRW. Loss of heterozygosity on chromosome 16p13.3 in hamartomas from tuberous sclerosis patients. Nature Genet 1994; 6: 193-196. 